\title{
Effectiveness of Repair of Aortic Coarctation in Neonates: A Long-Term experience
}

\author{
Chiara Minotti $^{1}$ (1) $\cdot$ Manuela Scioni $^{2} \cdot$ Biagio Castaldi $^{1} \cdot$ Alvise Guariento $^{3,4} \cdot$ Roberta Biffanti $^{1} \cdot$ Giovanni Di Salvo $^{1}$. \\ Vladimiro Vida ${ }^{3} \cdot$ Massimo A. Padalino $^{3}$
}

Received: 29 April 2021 / Accepted: 23 July 2021 / Published online: 2 August 2021

(c) The Author(s) 2021

\begin{abstract}
To evaluate early and long-term results of surgical treatment of aortic coarctation (CoAo) in neonates. This is a retrospective clinical review of neonates with CoAo, who underwent surgery between 1995 and 2019. Data were retrieved from our institutional database, to identify preoperative and postoperative characteristics. Statistical analysis was performed by means of relative risk ratio and Cox and logistic multivariate analysis. 218 consecutive neonates (M/F: 129/89, median age 11 days, IQR 7-17 days) were included; 202 (92.7\%) had a left thoracotomy; 178 underwent extended end-to-end anastomosis (EEEA, 81.6\%). Hypoplastic aortic arch (HAA) was present in 102 patients (46.8\%); complex cardiac anomalies in $85(39 \%)$. Significant postoperative complications occurred in $20(9.2 \%)$. Thirty-day mortality was $2.3 \%$ (most in complex types). At a median follow-up of 10.4 years (IQR 5.6-15.0 years; FU completeness 95.9\%), there were 8 late deaths (3.7\%), all associated to complex CoAo. Among 196 survivors, 177 (93.2\%) were in NYHA class I; re-interventions on aortic arch occurred in $9.2 \%$ (2.0\% were surgical). Freedom from mortality and re-intervention on aorta at 10 years were $94.3 \%$ and 96.7\%, respectively. Surgical repair of CoAo in newborns without CPB in our series was safe and low-risk, with excellent early and late outcomes.
\end{abstract}

Keywords Aortic coarctation $\cdot$ Neonates $\cdot$ Surgical repair $\cdot$ Outcome

\section{Introduction}

Aortic coarctation (CoAo) is a common congenital heart disease (CHD), occurring in approximately 4 out of 10,000 live births [1,2], which may be isolated or complex, when associated with other CHDs, such as a bicuspid aortic valve

This paper was presented at the CHSS\&ECHSA Joint Meeting, Boston October 24th and 25th, 2020.

Chiara Minotti

minotti.chiara@gmail.com

1 Pediatric Cardiology, Department of Women's and Children's Health, University of Padova, Via Giustiniani 2, 35128 Padova, Italy

2 Department of Statistical Sciences, University of Padova, Padova, Italy

3 Pediatric and Congenital Cardiac Surgery, Department of Cardiothoracic and Vascular Sciences and Public Health, University of Padova, Padova, Italy

4 Division of Cardiovascular Surgery, Department of Surgery, Hospital for Sick Children, Toronto, Canada
(BAV), ventricular septal defect (VSD), and hypoplastic aortic arch (HAA) [3]. In particular, HAA repair in either isolated or complex aortic coarctation may be associated with significant morbidity $[4,5]$.

Surgical planning is influenced by the need for concomitant repair of associated CHD [6]. In particular, the presence of HAA can be determinant for choosing the surgical approach (i.e., sternotomy vs. thoracotomy) or use of cardio-pulmonary bypass (CPB), deep hypothermic circulatory arrest (DHCA) [7], or selective antegrade cerebral perfusion (ACP) $[5,8]$.

Since the first report of the end-to-end anastomosis (EEA) by Crafoord in 1945 [9], several techniques have been proposed, such as aortic isthmoplasty [10], subclavian flap aortoplasty [11], extended end-to-end anastomosis (EEEA) [12], end-to-side anastomosis (ESA), and patch/conduit repair. Currently, the optimal surgical approach for CoAo repair in neonates, especially when associated with HAA, is controversial. Undoubtedly, an EEEA through a thoracotomy avoids $\mathrm{CPB}$ and the risk of adverse events (AE) related to DHCA or selective ACP $[6,13,14]$. However, a possible 
disadvantage is leaving the proximal HAA untreated, with a potential need of a future re-intervention.

Numerous studies have explored postoperative outcomes [8, 14-16]. Nevertheless, they had a relatively short follow-up, were limited to one surgical approach, or included patients undergoing surgical repair at different ages.

In this study, we reviewed our experience with neonatal repair of CoAo, to assess early and late morbidity, mortality, and re-intervention rate, with particular attention to late clinical outcomes.

\section{Methods}

This is a single-center, retrospective clinical study including all consecutive neonates ( $<30$ days of age) undergoing surgical repair from January 1995 to December 2019. A review of medical records was approved by our Hospital Committee on clinical investigation (4451/AO/18). Individual patients were not identified, and the need for patient consent was waived. Demographic, operative, and short-term outcomes included preoperative characteristics, any intervention (surgery or balloon dilation) before CoAo repair (also defined pre-CoAo procedures), associated anomalies, type of repair and surgical approach (thoracotomy vs sternotomy), associated surgical procedures, use of $\mathrm{CPB}, \mathrm{ACP}$, or DHCA, onset of major postoperative complications, and early ( $<30$ days) death. CoAo repair was defined "Complex" when associated with major CHD.

Preoperative echocardiographic images were evaluated by 2 different cardiologists, who were unaware of the original echocardiographic report and clinical outcomes. The dimensions of proximal (PAA between innominate and left carotidLCA-arteries) and distal aortic arch (DAA, between LCA and left subclavian artery) were measured and indexed by body surface area; we defined HAA when $Z$-scores of PAA and/or DAA were $<$ than -3 , as elsewhere stated [15].

Follow-up (FU) data included clinical status (NYHA class); late ( $>30$ days) death, re-interventions, surgical and/ or catheterization procedures, either cardiac or specifically on the previously repaired aortic site; arterial hypertension requiring medical treatment; aortic aneurysm; aortic valve dysfunction. All these events might have occurred in the same patient. In particular, clinical FU evaluation included a complete 2D Echocardiography to evaluate the peak and medium pressure gradient and the presence of diastolic runoff at the isthmus level with continuous Doppler and abdominal aorta pulsed wave Doppler to study the wave shape and the presence of diastolic tail. A recoarctation was defined as the presence of a superior-inferior limb arterial pressure gradient $>20 \mathrm{mmHg}$ at rest, with or without a mean isthmic pressure gradient $>20 \mathrm{mmHg}$, and a diastolic tail in pulsed wave Doppler in the abdominal aorta.

\section{Surgical Technique}

Most commonly, on right lateral decubitus, a left mini-thoracotomy was made in the 4th intercostal space. After lung retraction, the stenotic aortic isthmus was exposed. The ductus was suture-ligated and resected, with extensive mobilization of the distal aorta (at least $10 \mathrm{~mm}$ below the isthmus). The PAA was extensively mobilized until the LCA or to the innominate artery, to enhance adequate exposure of the entire PAA to the ascending aorta. The aortic isthmus was resected, removing all residual ductal tissue, to avoid late scar tissue retraction. The proximal incision was extended in the concavity of the aortic arch to the origin of the LCA or the innominate artery. Subsequently, both segments were re-approximated in a beveled fashion, and the anastomosis was performed with a continuous 7.0 prolene suture. Repair was considered optimal if the residual upper-lower limbs pressure gradient is $\leq 10 \mathrm{~mm} \mathrm{Hg}$.

\section{Statistical Analysis}

Quantitative variables were presented as median \pm interquartile range (IQR), while categorical variables were reported as frequency and percentage. A bivariate analysis was performed to assess the effect of a single predictive variable on outcomes; the association with outcomes as early and late death, any reintervention on aorta, and hypertension, and each predictive variable was measured using the relative risk (RR) and related 95\% confidence interval (CI). Risk factors were gender, age class ( $<15$ days, $\geq 15$ days) at surgery, urgent surgery, BAV, hypoplastic LV/single ventricle, pre-CoAo procedures, HAA, complex CoAo, EEEA vs other procedures, CPB, thoracotomy, and associated surgical procedures.

Survival analysis was performed by Kaplan-Meier curves and log-rank test. Multivariate models were applied using Cox proportional hazards for outcomes (death, re-intervention on aorta). Significant risk factors in the bivariate analysis were included in the multivariate models. Multivariate logistic regression was performed to analyze systemic hypertension. The significance level was set at 0.05 , and all tests were twotailed. The inverse probability of treatment weighting (IPTW) was applied to minimize the fact that the surgical procedure was not assigned randomly to the patients, and it was based on the covariates mentioned above. Data were analyzed using $\mathrm{R}$ version 2.6.2.

\section{Results}

We included 218 consecutive neonates (M/F: 129/89), with a median age at surgery of 11 days (IQR 7-17 days). CoAo was isolated in 133 (61.0\%); 102 (46.8\%) had HAA. All preoperative data are listed in Table 1. Most patients underwent 
EEEA $(178,81.6 \%)$. Complete preoperative echocardiographic data were available in 40 patients. Median PAA Z-score was -4.1 (IQR -6.1 to -2.7 ), while median DAA Z-score was -3.2 (IQR -4.1 to -2.2$)$. A left lateral thoracotomy was used in 202 patients $(92.7 \%)$. Associated procedures were performed in 61 patients (27.9\%). Prior to CoAo repair, 8 patients required intervention (balloon dilation in 6 , pulmonary artery banding and atrioseptostomy in 2 , respectively). Significant postoperative complications occurred in 20 patients $(9.2 \%$, Table 2$)$. There were no early reoperations. Thirty-day mortality was $2.3 \%$ (5 patients, 3 of whom with complex CoAo, after repair on CPB). All intraoperative and postoperative data are summarized in Table 2.

\section{Follow-Up}

Late clinical outcomes are summarized in Table 2. At a median FU of 10.4 years (IQR 5.6-15.0 years; completeness $95.9 \%)$, there were $8(3.7 \%)$ late deaths in the complex group. Among 196 survivors (89.9\%), re-interventions on aortic arch occurred in $18(9.2 \%)$, but only 4 patients (1.8\%) needed a surgical reoperation ( 3 in complex CoAo), while the remaining were effectively treated with balloon angioplasty. Among survivors, 183 (93.4\%) were in NYHA class I and $18(9.2 \%)$ were on anti-hypertensive medical treatment. However, all patients were presented at FU with a normal abdominal aorta pulsed wave Doppler ultrasound and a superior-inferior limbs arterial pressure $<20 \mathrm{mmHg}$ at rest.

Bivariate analysis (Table 3a) showed that the presence of hypoplastic LV, HAA, or complex CoAo significantly affected survival. Conversely, patients undergoing EEEA had a significantly lower risk of re-intervention, showing better outcomes (RR 0.385, $p=0.048$ ) (Table 3b). Pre-CoAo correction procedures, any surgical technique other than EEA and EEEA, use of CPB, complex CoAo, associated surgical procedures, and urgent operation were significant risk factors for late hypertension (Table 3c).

Logistic regression confirmed that pre-CoAo procedures, urgent repair, and sternotomy were associated with late onset of arterial hypertension (Table 4). After IPTW, the logistic regression confirmed that the type of surgical procedure was affecting signfiicantly the risk for late arterial hypertension (Table 5). Also, in the subgroup with available measurements, a $Z$-score $<-3$ of PAA, DAA, and isthmus did not result statistically significant for development of late arterial hypertension ( $p=0.459, p=0.310, p=0.6$, respectively).

Multivariate analysis at Cox's regression model (Table 6) showed that only a hypoplastic LV was a significant risk factor for mortality (HR 18.74, $p<0.001$ ), while HAA was not significantly associated with reoperation $(p=0.363)$. As far as risk for late re-intervention on aorta is concerned, we included control variables other than EEEA (HAA, thoracotomy vs sternotomy approach, complex vs simple CoAo) in the multivariate Cox model, and we observed a tendency of EEEA to protect from re-intervention $(p=0.09)$. However, after IPTW, the type of surgical procedure (EEEA vs EEA and other) was not significantly affecting mortality and re-intervention on aorta (Table 7).

Last, freedom from mortality (Fig. 1) and re-intervention on aorta (Fig. 2) were $94.3 \%$ and $96.7 \%$, respectively, at a median FU of 10.4 years.

\section{Comment}

In this retrospective study, repair of neonatal CoAo was performed mostly by EEEA through thoracotomy, with a very low postoperative morbidity and mortality. Also, freedom from re-intervention for late recoarctation was $96.7 \%$ at a median FU of 10.4 years, which, to the best of our knowledge, is the longest ever reported [15-17]. Our experience

Table 1 Preoperative data

\begin{tabular}{|c|c|c|c|c|}
\hline & Total: 218 & Isolated CoAo type: 133 (61.0) & Complex CoAo type: 85 (39.0) & $p$ value \\
\hline Male $(n, \%)$ & $129 / 218(59.2)$ & $81 / 129(62.8)$ & $48 / 129(37.2)$ & $p=0.516149$ \\
\hline Median age at surgery & 11 days (IQR 7-17) & 11 days (IQR 8-16) & 12 days (IQR 7-18) & NA \\
\hline \multicolumn{5}{|l|}{ Age category $(n, \%)$} \\
\hline$<15$ days & $142(65.1)$ & $93 / 142(65.5)$ & $49 / 142(34.5)$ & \multirow[t]{2}{*}{$p=0.195417$} \\
\hline$\geq 15$ days & $76(34.9)$ & $43 / 76(56.5)$ & $33 / 76(43.5)$ & \\
\hline HAA $(n, \%)$ & $102(46.8)$ & $41 / 102(40.0)$ & $61 / 102(60.0)$ & $p=0.00001$ \\
\hline $\operatorname{BAV}(n, \%)$ & $65(29.8)$ & 37 & 28 & $p=0.420099$ \\
\hline Hypoplastic LV $(n, \%)$ & $11(5.1)$ & $0 / 11(0)$ & $11 / 11(100)$ & NA \\
\hline Single ventricle $(n, \%)$ & $1(0.5)$ & $0 / 1(0)$ & $1 / 1(100)$ & NA \\
\hline Pre-CoAo surgical intervention $(n, \%)$ & $2(0.9)$ & $1 / 2(50)$ & $1 / 2(50)$ & $p=1$ \\
\hline Pre-CoAo balloon dilation $(n, \%)$ & $6(2.8 \%)$ & $6 / 6(100)$ & $0 / 6(0)$ & NA \\
\hline
\end{tabular}

Significant $p$ value is given in bold

$B A V$ bicuspid aortic valve, CoAo aortic coarctation, $H A A$ hypoplastic aortic arch, $L V$ left ventricle, $N A$ not applicable 
Table 2 Early and late outcomes

\begin{tabular}{|c|c|c|c|c|}
\hline & Total 218 & $\begin{array}{l}\text { Isolated CoAo } \\
\text { type:133 (61.0) }\end{array}$ & $\begin{array}{l}\text { Complex CoAo } \\
\text { type: } 85 \text { (39.0) }\end{array}$ & $p$ value \\
\hline \multicolumn{5}{|l|}{ Surgical intervention } \\
\hline $\operatorname{EEEA}(n, \%)$ & 178 (81.6) & $104 / 178(58.4)$ & 74/178 (41.6) & $p=0.099148$ \\
\hline $\operatorname{EEA}(n, \%)$ & $35(16.1)$ & $18 / 35(51.4)$ & $17 / 35(48.6)$ & $p=0.204661$ \\
\hline Other $(n, \%)^{\mathrm{a}}$ & $5(2.3)$ & $4 / 5(80)$ & $1 / 5(20)$ & $p=0.378414$ \\
\hline Operative approach & & & & $p=0.000036$ \\
\hline Lateral thoracotomy $(n, \%)$ & $202(92.7)$ & $131 / 202(64.8)$ & $71 / 202(35.2)$ & \\
\hline Median sternotomy and CPB $(n, \%)$ & $16(7.3)$ & $2 / 16(12.5)$ & $14 / 16(87.5)$ & \\
\hline Associated surgical procedures $(n, \%)$ & $61(27.9)$ & $16 / 61(26.2)$ & 45/61 (73.8) & $p<0.00001$ \\
\hline Urgent procedure $(n, \%)$ & $23(10.5)$ & $11 / 23(47.8)$ & $12 / 23(52.2)$ & $p=0.170497$ \\
\hline \multicolumn{5}{|l|}{ Early outcomes } \\
\hline Postoperative complications $(n, \%)$ & $20(9.2)$ & $9 / 20(45)$ & $11 / 20(55)$ & $p=0.123491$ \\
\hline Heart failure $(n)$ & 5 & $3 / 5(60)$ & $2 / 5(40)$ & \\
\hline Respiratory failure $(n)$ & 3 & $1 / 3(33.3)$ & $2 / 3(66.7)$ & \\
\hline Infections/sepsis $(n)$ & 3 & $0 / 3(0)$ & $3 / 3(110)$ & \\
\hline Arrhythmias $(n)$ & 2 & $2 / 2(100)$ & $0 / 2(0)$ & \\
\hline Renal failure $(n)$ & 2 & $1 / 2(50)$ & $1 / 2(50)$ & \\
\hline Pulmonary hypertension $(n)$ & 1 & $0 / 1(0)$ & $1 / 1(100)$ & \\
\hline Other $(n)$ & 4 & $2 / 4(50)$ & $2 / 4(50)$ & \\
\hline Need for re-intervention $(n, \%)$ & $0(0)$ & & & NA \\
\hline Thirty-day mortality $(n, \%)$ & $5(2.3)$ & $2 / 133(1.5)$ & $3 / 85(3.5)$ & $p=0.32984$ \\
\hline \multicolumn{5}{|l|}{ Late outcomes } \\
\hline Alive with follow-up $(n, \%)$ : & $196(89.9)$ & $125 / 196(64.7)$ & $71 / 196(35.3)$ & $p=\mathbf{0 . 0 1 2 4 3 3}$ \\
\hline Lost to follow-up $(n, \%)$ : & $9(4.1)$ & $6 / 9(66.6)$ & $3 / 9(33.4)$ & $p=0.722287$ \\
\hline Median follow-up time (years, IQR) & $10.4(5.6-15.0)$ & $10.6(6.7-16.5)$ & $10.2(1.3-14.9)$ & $p=0.08726$ \\
\hline Late mortality $(n, \%)$ : & $8(3.7)$ & 0 & 8 & $p=000,757$ \\
\hline Overall mortality $(n, \%)$ : & $13(6.0)$ & 2 & 11 & $p=0.000505$ \\
\hline Re-intervention on aorta $(n, \%)$ & $18(9.2)$ & $8 / 18$ & $10 / 18$ & $p=0.132486$ \\
\hline Balloon dilation $(n, \%)$ & $14(7.4 \%)$ & 7 & 7 & $p=1$ \\
\hline Surgical re-intervention $(n, \%)$ & $4(1.8 \%)$ & 1 & 3 & $p=0.136135$ \\
\hline \multicolumn{5}{|l|}{ NYHA class } \\
\hline $\mathrm{I}(n, \%)$ & $183(93.4)$ & $120 / 183(65.6)$ & $63 / 183(34.4)$ & $p=0.001579$ \\
\hline II $(n, \%)$ & $12(6.1)$ & $5 / 12(41.6)$ & $7 / 12(58.4)$ & $p=0.166061$ \\
\hline III $(n, \%)$ & $0(0)$ & $0(0)$ & $0(0)$ & NA \\
\hline $\mathrm{IV}(n, \%)$ & $1(0.5)$ & $0 / 1(0)$ & $1 / 1(100)$ & NA \\
\hline Anti-hypertensive treatment $(n, \%)$ & $18(9.2)$ & 2/18 (11.1) & 16/18 (88.8) & $p<0.00001$ \\
\hline
\end{tabular}

Significant $p$ values are given in bold

$C P B$ cardio-pulmonary bypass, $C o A o$ aortic coarctation, EEEA extended end-to-end anastomosis, EEA end-to-end anastomosis, NA not applicable, NYHA New York Heart Association

${ }^{a}$ Other: aortic arch reconstruction with patch plasty and termino-lateral anastomosis shows that nowadays an effective neonatal repair of CoAo has excellent long-term outcomes.

\section{Surgical Technique}

Currently, EEEA is considered to be the most effective and safest approach even in patients with HAA [17]. More than $80 \%$ of our patients underwent surgical repair through EEEA (Table 2). In our hands, it had a good safety profile, with an overall operative mortality as low as $2.3 \%$, as confirmed also by other experiences [17], and with excellent late outcomes (RR 0.385, $p=0.048$ ), as demonstrated by the meager re-intervention rate on the aortic arch at long-term followup. In our series, EEEA showed a tendency to protect from late recoarctation and late re-intervention on aortic arch $(p=0.09$, Table 6), even if it was not confirmed after IPTW. Undoubtedly, EEEA has many advantages, such as complete resection of CoAo and ductal tissue, immediate access for 
Table 3 Bivariate analysis

\begin{tabular}{|c|c|c|c|c|c|}
\hline & $\mathrm{RR}$ & Lower & Upper & $p$-value & $n$ \\
\hline \multicolumn{6}{|l|}{ a. RR for early or late death } \\
\hline Sex (female) & 2.319 & 0.784 & 6.858 & 0.148 & 218 \\
\hline Age $\leq 15 \mathrm{gg}$ & 0.856 & 0.290 & 2.527 & 0.771 & 218 \\
\hline Bicuspid aortic valve & 0.428 & 0.098 & 1.877 & 0.353 & 218 \\
\hline Hypoplastic LV & 16.13 & 6.52 & 39.92 & $<0.001$ & 218 \\
\hline HAA & 13.65 & 1.81 & 103.14 & $<0.001$ & 218 \\
\hline Isolated vs complex & 8.606 & 1.955 & 37.875 & $<0.001$ & 218 \\
\hline \multicolumn{6}{|l|}{ Surgical procedure } \\
\hline EEEA & 0.590 & 0.168 & 2.070 & 0.421 & 218 \\
\hline Other than EEA/EEEA & 2.333 & 0.297 & 18.309 & 0.427 & \\
\hline Sternotomy and cardio-pulmonary bypass & 3.788 & 1.157 & 12,394 & 0.059 & 218 \\
\hline Thoracotomy & 0.264 & 0.081 & 0.864 & 0.059 & 218 \\
\hline Associated surgery & 1.609 & 0.548 & 4.725 & 0.360 & 218 \\
\hline Urgent procedure & 0.782 & 0.107 & 5.718 & 1 & 218 \\
\hline \multicolumn{6}{|l|}{ b. RR for re-intervention on aorta } \\
\hline Sex (female) & 1.263 & 0.522 & 3.058 & 0.619 & 196 \\
\hline Age class at surgery $(\leq 15 \mathrm{gg})$ & 1.350 & 0.503 & 3.628 & 0.613 & 196 \\
\hline Bicuspid aortic valve & 2.015 & 0.840 & 4.834 & 0.122 & 196 \\
\hline Hypoplastic LV & - & - & - & 1 & 196 \\
\hline Pre-CoAo procedure & 2.506 & 0.98 & 6.406 & 0.079 & 196 \\
\hline HAA & 1.879 & 0.804 & 4.393 & 0.159 & 196 \\
\hline Isolated vs complex & 2.106 & 0.871 & 5.095 & 0.124 & 196 \\
\hline \multicolumn{6}{|l|}{ Surgical procedure } \\
\hline EEEA & 0.385 & 0.156 & 0.949 & 0.048 & 196 \\
\hline Other than EEA/EEEA & 0 & 0 & - & 0.553 & \\
\hline Sternotomy and cardio-pulmonary bypass & 1.917 & 0.497 & 7.386 & 0.303 & 176 \\
\hline Thoracotomy & 0.522 & 0.135 & 2.011 & 0.303 & 176 \\
\hline Associated surgical procedures & 1.591 & 0.650 & 3.896 & 0.411 & 176 \\
\hline Urgent procedure & 0 & 0 & $\mathrm{Na}$ & 0.225 & 176 \\
\hline \multicolumn{6}{|l|}{ c. RR for late onset of Hypertension } \\
\hline Sex (female) & 0.602 & 0.224 & 1.621 & 0.447 & 194 \\
\hline Age class at surgery $(\leq 15 \mathrm{gg})$ & 0.523 & 0.218 & 1.256 & 0.192 & 194 \\
\hline Bicuspid aortic valve & 1.000 & 0.393 & 2.544 & 1 & 194 \\
\hline Hypoplastic LV & 2.809 & 0.485 & 16.277 & 0.323 & 194 \\
\hline Pre-CoAo procedure & 6.679 & 2.835 & 15.731 & 0.003 & 194 \\
\hline HAA & 1.267 & 0.526 & 3.055 & 0.626 & 194 \\
\hline Isolated vs complex & 8.356 & 2.504 & 27.888 & $<0.001$ & 194 \\
\hline Single ventricle & 0 & 0 & $\mathrm{Na}$ & 1 & 194 \\
\hline \multicolumn{6}{|l|}{ Type of surgical procedure } \\
\hline EEEA & 1.259 & 0.299 & 5.306 & 1 & 194 \\
\hline Other & 11.625 & 2.713 & 49.806 & 0.006 & 194 \\
\hline Sternotomy and cardio-pulmonary bypass & 7.625 & 3.471 & 16.749 & $<0.001$ & 194 \\
\hline Thoracotomy & 0.131 & 0.060 & 0.288 & $<0.001$ & 194 \\
\hline Associated surgical procedures & 4.964 & 1.959 & 12.577 & $<0.001$ & 194 \\
\hline Urgent procedure & 3.365 & 1.339 & 8.460 & 0.024 & 194 \\
\hline
\end{tabular}

CoAo aortic coarctation, EEA end-to-end anastomosis, EEEA extended end-to-end anastomosis, $H A A$ hypoplastic aortic arch, $L V$ left ventricle, $R R$ risk ratio 
Table 4 Logistic regression for hypertension

\begin{tabular}{lccccc}
\hline & Estimate & Std error & $z$-value & $p$-value & $n$ \\
\hline Pre-CoAo correction Interventions & 2.7945 & 0.9318 & 2.999 & 0.00271 & 194 \\
Associated surgical procedures & 0.9487 & 0.6619 & 1.433 & 0.15181 & 194 \\
Urgent surgery & 1.7193 & 0.7488 & 2.296 & 0.02166 & 194 \\
Complex CoAo & 1.1632 & 0.7613 & 1.528 & 0.12654 & 194 \\
Sternotomy & 2.1531 & 0.8996 & 2.393 & 0.01669 & 194 \\
Type of repair EEEA vs EEA & 1.3673 & 1.0635 & 1.286 & 0.19856 & 194 \\
Type of repair other vs EEA & 2.8298 & 1.5879 & 1.782 & 0.07473 & 194 \\
Intercept & -3.4603 & 1.4151 & -2.445 & 0.01447 & 194 \\
\hline
\end{tabular}

$E E A$ end-to-end anastomosis, EEEA extended end-to-end anastomosis, $S t d$ standard, $R R$ risk ratio
Table 5 Logistic regression for hypertension after inverse probability weighting

\begin{tabular}{lccccc}
\hline & Estimate & Std error & $z$-value & $p$-value & $n$ \\
\hline $\begin{array}{l}\text { Type of repair } \\
\text { EEEA vs other }\end{array}$ & 0.5579 & 0.6623 & 0.842 & 0.4006 & 194 \\
Intercept & -5.4696 & 0.8034 & -6.808 & $<0.001$ & 194 \\
\hline
\end{tabular}

$E E A$ end-to-end anastomosis, EEEA extended end-to-end anastomosis, Std standard, $R R$ risk ratio

Table 6 Multivariate analysis: Cox's regression model

\begin{tabular}{|c|c|c|}
\hline \multirow[t]{2}{*}{ Variables } & \multicolumn{2}{|c|}{ Outcomes HR (CI 95\%) $p$ value } \\
\hline & Death & Re-intervention on aorta \\
\hline Hypoplastic LV & $\begin{array}{l}18.74(5.43-64.7) \\
p<0.001\end{array}$ & - \\
\hline Thoracotomy & $\begin{array}{l}0.39(0.10-1.6) \\
p=0.184\end{array}$ & $\begin{array}{l}0.75(0.16-3.5) \\
p=0.718\end{array}$ \\
\hline HAA & $\begin{array}{l}7.68(0.94-62.8) \\
p=0.057\end{array}$ & $\begin{array}{l}1.68(0.55-5.2) \\
p 0.363\end{array}$ \\
\hline Complex CoAo & $\begin{array}{l}2.12(0.41-11.0) \\
p=0.371\end{array}$ & $\begin{array}{l}1.85(0.64-5.4) \\
p=0.259\end{array}$ \\
\hline $\begin{array}{l}\text { EEEA vs other tech- } \\
\text { niques }\end{array}$ & - & $\begin{array}{l}0.43(0.6-1.2) \\
p=0.093\end{array}$ \\
\hline
\end{tabular}

EEEA extended end-to-end anastomosis, $H R$ hazard ratio, $L V$ left ventricle, $H A A$ hypoplastic aortic arch, CoAo aortic coarctation

Table 7 Multivariate analysis: Cox's regression model after inverse probability weighting

\begin{tabular}{lll}
\hline Variables & $\begin{array}{l}\text { Outcomes HR (CI 95\%) } \\
p \text { value }\end{array}$ \\
\cline { 2 - 3 } & Death & Re-intervention on aorta \\
\hline EEEA vs other tech- & $\begin{array}{l}0.810(0.2,2.9) \\
\text { niques }\end{array}$ & $\begin{array}{l}0.47(0.16-1.4) \\
p=0.745\end{array}$ \\
\hline
\end{tabular}

EEEA extended end-to-end anastomosis, $H R$ hazard ratio, $L V$ left ventricle, $H A A$ hypoplastic aortic arch, CoAo aortic coarctation hypoplasia of transverse arch, and preservation of left subclavian artery. Moreover, it does not require use of prosthetic tissue and can be performed either through left thoracotomy or sternotomy. As noted elsewhere [18], extensive proximal and distal tissue dissection are of paramount importance to mobilize the elastic neonatal aorta and brachiocephalic vessels, and to allow adequate exposure of PAA to the ascending aorta, and an effective aortic arch reconstruction without the drawbacks of CPB or DHCA. Moreover, an extensive dissection may prevent from late scar tissue retraction and can reduce recoarctation rate, and it was found to be safe, with no related complications (such as chylothorax) in our experience [8].

\section{Hypoplastic Aortic Arch}

Several studies reinforced the importance of identifying cut points that could help the surgeon to decide the most effective approach to achieve optimal repair with a low risk of late re-intervention for residual aortic arch hypoplasia [19, 20]. Kotany [15] reported that despite severe PAA stenosis ( $z$-value $<-6$ ), EEEA still had a $90 \%$ freedom from reoperation at 3 years. Tulzer [19] identified a PAA cut-off $z$-value of -4.50 for a safe EEEA and a freedom from re-intervention of $90.12 \%$ at 10 years. According to Gropler [17], a $z$-value lower than -4.1 for PAA and lower than -2.8 for DAA may accurately predict the candidate selection for median sternotomy with a good sensitivity profile. Similarly, we collected echocardiographic measurements of PAA and DAA. However, due to insufficient data, we could not recognize useful cut-off values, since none of the 40 patients with available measurements had late recoarctation. Even if we cannot demonstrate whether hypoplastic PAA rather than DAA can cause recoarctation, in our neonatal series we had a very low re-intervention rate $(9.2 \%)$ at a very long median follow-up of 10.4 years, which is longer than in other recent reports [17].

However, although minimized, the problem of recoarctation still exists. Transverse aortic arch hypoplasia and tubular hypoplasia are typically associated with intracardiac defects, 


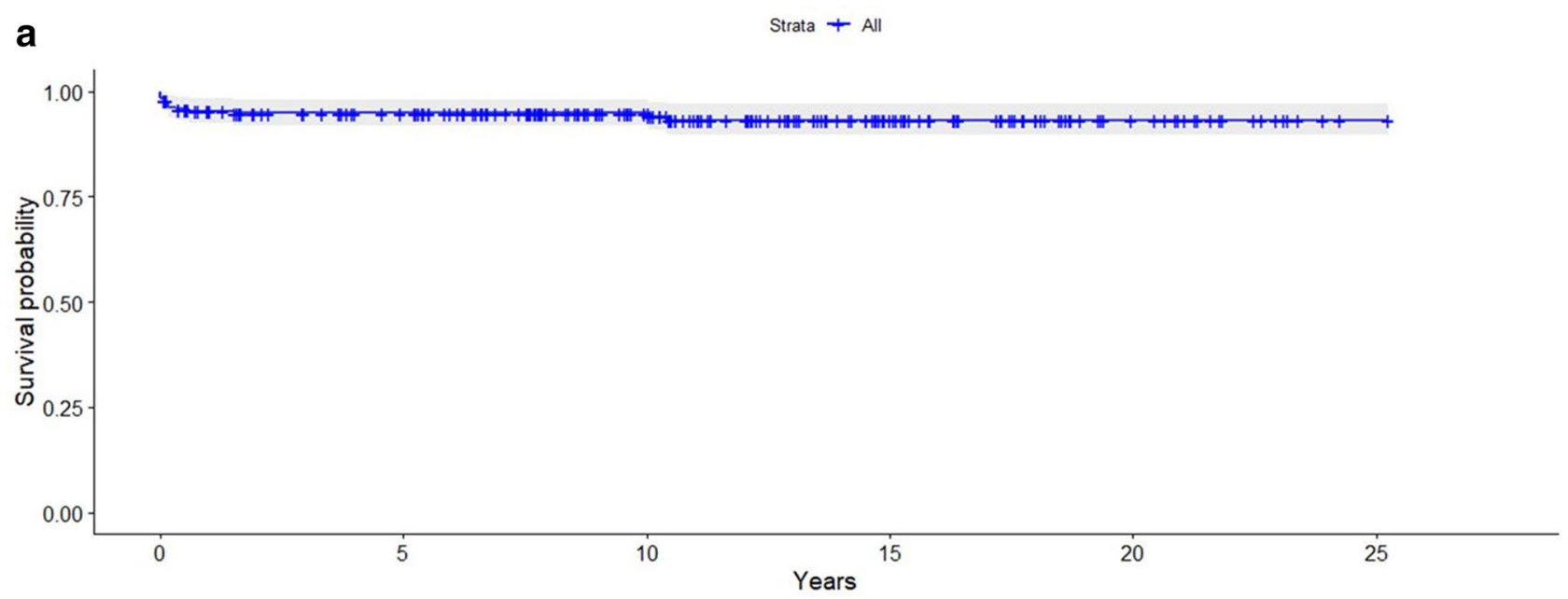

\begin{tabular}{|c|c|c|c|c|c|c|}
\hline 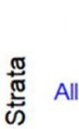 & $\begin{array}{l}\text { umber } \\
218\end{array}$ & 170 & 117 & 54 & 19 & 1 \\
\hline & 0 & 5 & 10 & 15 & 20 & 25 \\
\hline
\end{tabular}

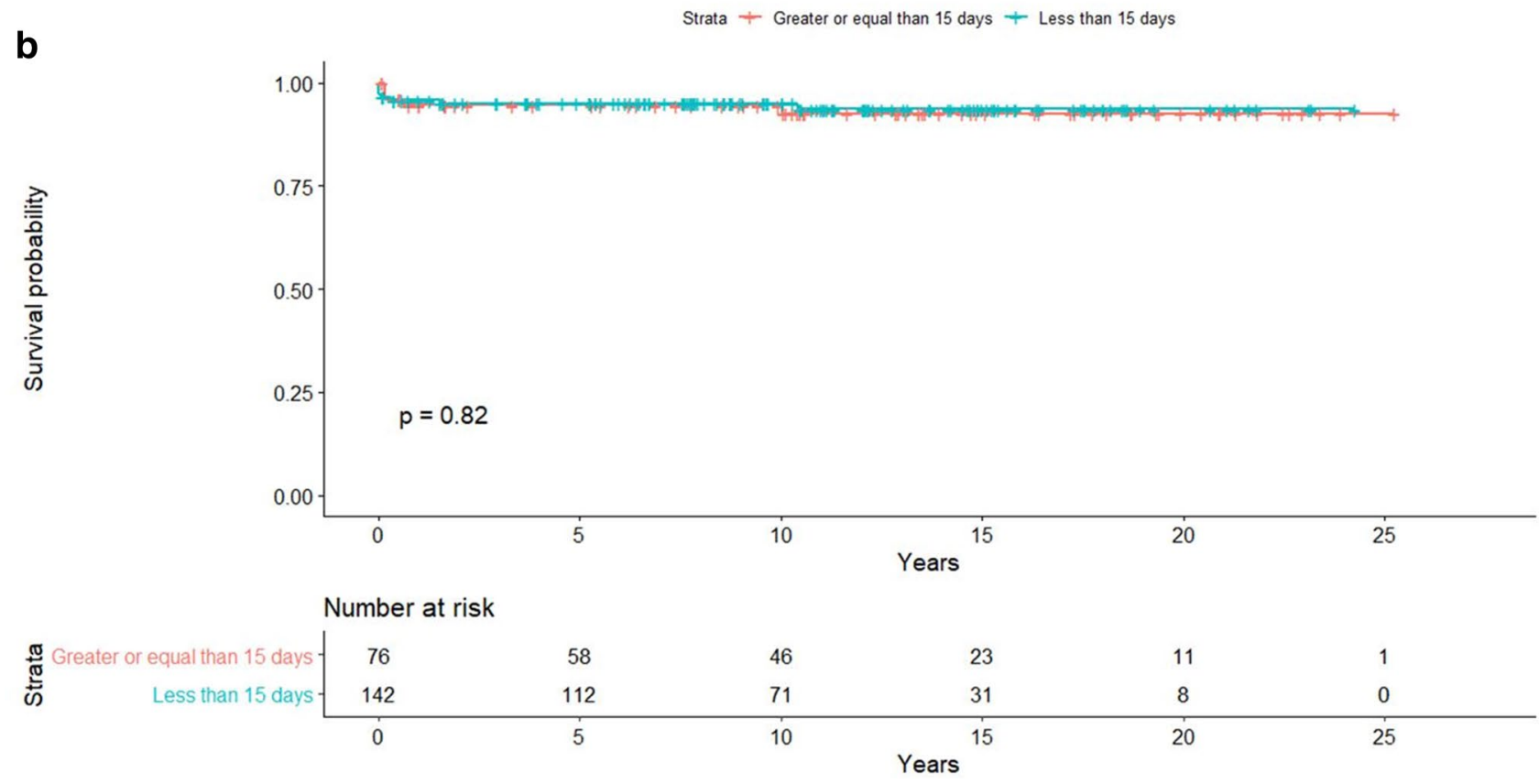

Fig. 1 Kaplan-Meier survival plot, showing freedom from mortality, overall (a) and according to age subgroup (b)

such as large VSD or hypoplastic left heart syndrome. Currently, transverse aortic arch is considered of an acceptable size if its $z$-value diameter is $>-3$, or if it is equal (in $\mathrm{mm}$ ) to patient's body weight +1 [7]. As stated elsewhere [11], a hypoplastic PAA with $z$-score $\geq-6$ involving ascending aorta may be corrected with good results by EEEA through thoracotomy. Conversely, severe hypoplastic PAA $(<-6)$ with or without hypoplastic ascending aorta should be corrected with better outcomes by midline sternotomy with CPB [7], to reduce the risk of leaving a residual gradient. On this basis, we currently choose sternotomy with $\mathrm{CPB}$ in case of associated complex CHD, extremely hypoplastic ascending aorta and PAA $(Z$-score $<-6)$, or in candidates to simultaneous VSD repair. 


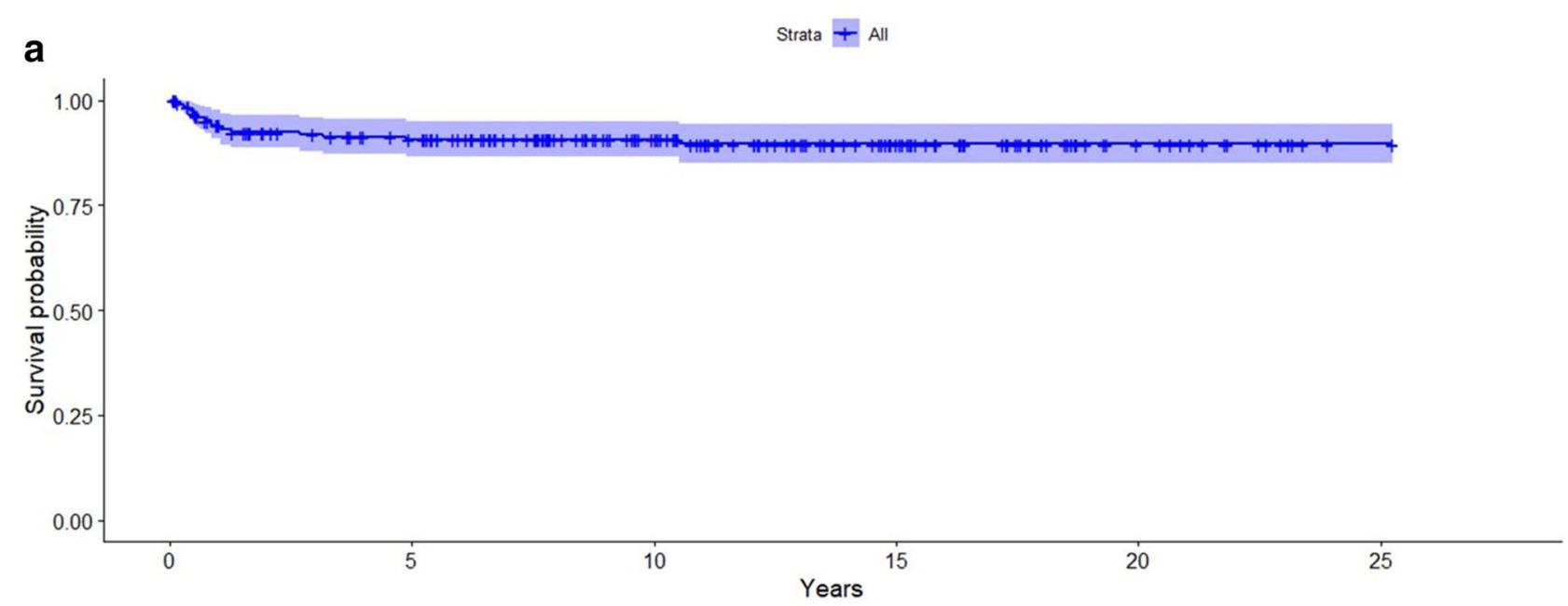

\begin{tabular}{|c|c|c|c|c|c|c|}
\hline All & mber & 143 & 94 & 48 & 15 & 1 \\
\hline & 0 & 5 & 10 & 15 & 20 & 25 \\
\hline
\end{tabular}

b

Strata + Greater or equal than 15 days + Less than 15 days

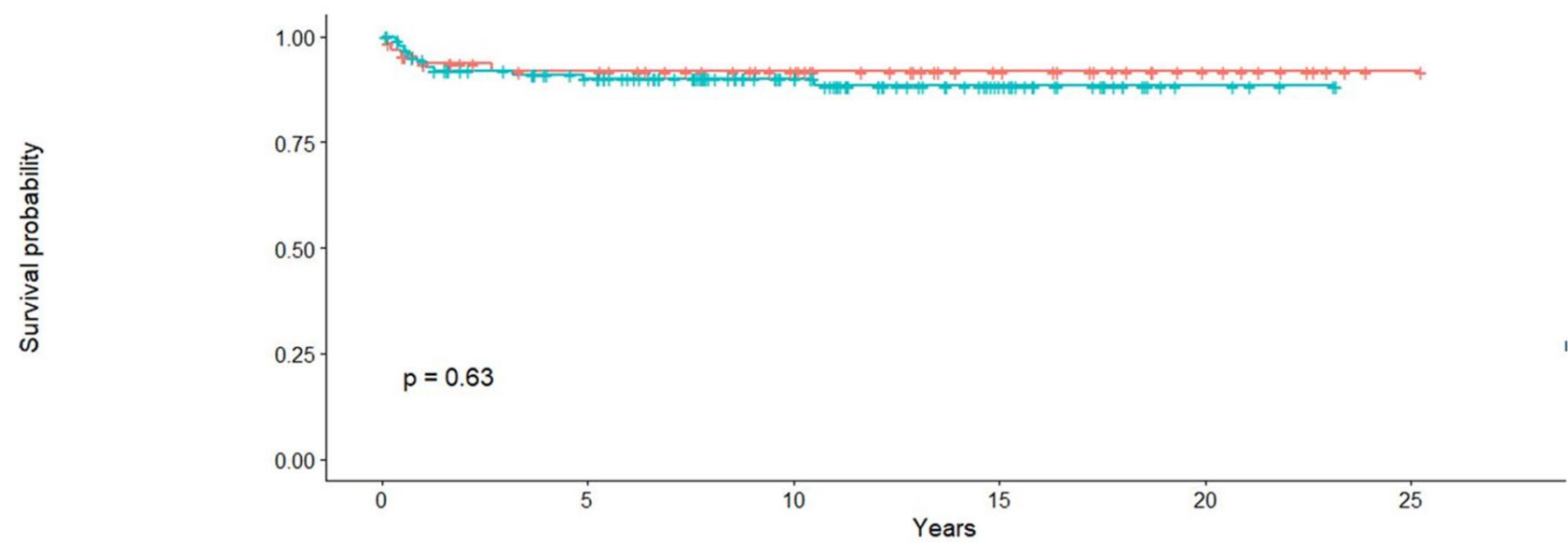

\begin{tabular}{|c|c|c|c|c|c|c|}
\hline \multirow{4}{*}{ 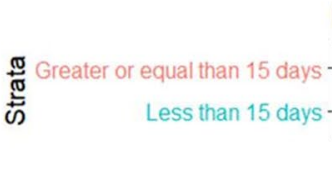 } & \multicolumn{6}{|c|}{ Number at risk } \\
\hline & 67 & 48 & 36 & 21 & 10 & 1 \\
\hline & 129 & 95 & 58 & 27 & 5 & 0 \\
\hline & 0 & 5 & 10 & 15 & 20 & 25 \\
\hline
\end{tabular}

Fig. 2 Kaplan-Meier survival plot, showing freedom from re-intervention, overall (a) and according to age subgroup (b)

\section{Late Arterial Hypertension}

Gropler et al. reported a prevalence of late hypertension of $18 \%$ [17]. Recently, Lee et al. [21] remarked a high prevalence $(59 \%)$ of late-onset hypertension after CoAo repair, with $37 \%$ of patients presenting recoarctation and late hypertension (OR 2.28). In that series, most patients had repair with a left subclavian flap (41\%), a prosthetic patch (11\%), or simple resection (2\%). Our IPTW analysis suggests that the repair technique (when well performed) may not affect significantly the onset of arterial hypertension. Age at repair is probably what makes the difference. In our series, arterial hypertension was significantly lower than other reports (9.2\%). Only 18 patients had medically treated hypertension and all had a normal abdominal aorta pulsed wave Doppler ultrasound at long-term FU. Our median age at repair was 
11 days, which might contribute to minimize the known risk factor for hypertension development that is older age [22].

\section{Lessons from our Experience}

In our experience, surgical repair of CoAo was mostly performed by the lateral approach through left thoracotomy. This approach has been favored since it avoids CPB and its related risks. This approach has been used also in cases of complex CoAo (with associated major intracardiac lesions), in the setting of a planned staged complete repair. Median sternotomy with CPB was generally preferred in case of complex CHD, with the need of multiple concomitant surgical procedures, or with severe PAA hypoplasia. This surgical strategy in our hands has been showing excellent early and late outcomes, and interestingly, we observed a low incidence of late re-interventions even when distal HAA was associated.

On this basis, and since balloon dilation was highly effective for late recoarctation treatment, we strongly advise for early CoAo repair by no-CPB approach through a left thoracotomy. Also, few patients are on anti-hypertensive therapy at follow-up, which demonstrates the effectiveness of the neonatal repair on reversing the hypertension physiopathological mechanism.

\section{Limitations}

Although our series presents one of the longest followup times in patients undergoing neonatal CoAo repair, an intrinsic limitation is the retrospective nature of the investigation, which covered more than two decades of surgical experience. This allowed the collection of precise echocardiographic measures of PAA and DAA in only 40 patients. Therefore we do not have enough data to discriminate between PAA and DAA impact on late recoarctation. Last, we could not collect enough data on late term stress test to evaluate hypertensive response on effort.

\section{Conclusion}

Surgical repair of CoAo in neonates by lateral approach without CPB is a safe and low-risk procedure, with excellent early and late outcomes. The incidence of late re-interventions is low. As the need for re-intervention on aortic arch has been rare in our series and balloon dilation was highly effective for late recoarctation treatment, neonatal repair of CoAo without CPB through a left thoracotomy is an optimal approach for a long-term effective treatment of CoAo, even when distal HAA is associated.
Funding Open access funding provided by Università degli Studi di Padova within the CRUI-CARE Agreement. This work received no funding.

Data Availability Upon request.

Code Availability Not applicable.

\section{Declarations}

Conflict of interest The authors declare that they have no conflict of interest.

Open Access This article is licensed under a Creative Commons Attribution 4.0 International License, which permits use, sharing, adaptation, distribution and reproduction in any medium or format, as long as you give appropriate credit to the original author(s) and the source, provide a link to the Creative Commons licence, and indicate if changes were made. The images or other third party material in this article are included in the article's Creative Commons licence, unless indicated otherwise in a credit line to the material. If material is not included in the article's Creative Commons licence and your intended use is not permitted by statutory regulation or exceeds the permitted use, you will need to obtain permission directly from the copyright holder. To view a copy of this licence, visit http://creativecommons.org/licenses/by/4.0/.

\section{References}

1. Baumgartner H, Bonhoeffer P, De Groot NMS et al (2010) ESC Guidelines for the management of grown-up congenital heart disease (new version 2010). Eur Heart J 31(23):2915-2957

2. Singh S, Hakim FA, Sharma A et al (2015) Hypoplasia, pseudocoarctation and coarctation of the aorta- a systematic review. Heart Lung Circ 24(2):110-118

3. Brown ML, Burkhart HM, Connolly HM et al (2013) Coarctation of the aorta: lifelong surveillance is mandatory following surgical repair. J Am Coll Cardiol 62(11):1020-1025

4. Marelli A, Miller SP, Marino BS, Jefferson AL, Newburger JW (2016) Brain in congenital heart disease across the lifespan: the cumulative burden of injury. Circulation 133(20):1951-1962

5. Luciani GB, Hoxha S, Angeli E et al (2019) Selective versus standard cerebro-myocardial perfusion in neonates undergoing aortic arch repair: a multi-center study. Artif Organs 43(8):728-735

6. Callahan CP, Saudek D, Creighton S et al (2019) Proximal arch in left thoracotomy repair of neonatal and infant coarctationhow small is too small? World J Pediatr Congenit Hear Surg 10(4):469-474

7. Karl TR, Sano S, Brawn W, Mee RB (1992) Repair of hypoplastic or interrupted aortic arch via sternotomy. J Thorac Cardiovasc Surg 104(3):688-695

8. Padalino MA, Bagatin C, Bordin G et al (2019) Surgical repair of aortic coarctation in pediatric age: a single center two decades experience. J Card Surg 34(5):256-265

9. Crafoord C, Nylin G (1945) Congenital coarctation of the aorta and its surgical treatment. Thorac Surg 14:347-361

10. Vossschulte K (1961) Surgical correction of coarctation of the aorta by an "isthmusplastic" operation. Thorax 16:338-345

11. Waldhausen JA, Nahrwold DL (1966) Repair of coarctation of the aorta with a subclavian flap. J Thorac Cardiovasc Surg 51(4):532-533 
12. Amato JJ, Rheinlander HFCR (1977) A method of enlarging the distal transverse arch in infants with hypoplasia and coarctation of the aorta. Ann Thorac Surg 23:261-263

13. Vedovelli L, Padalino M, D'Aronco S et al (2017) Glial fibrillary acidic protein plasma levels are correlated with degree of hypothermia during cardiopulmonary bypass in congenital heart disease surgery. Interact Cardiovasc Thorac Surg 24(3):436-442

14. Kornilov IA, Sinelnikov YS, Soinov IA et al (2015) Outcomes after aortic arch reconstruction for infants: deep hypothermic circulatory arrest versus moderate hypothermia with selective antegrade cerebral perfusion. Eur J Cardiothorac Surg 48(3):e45-50

15. Kotani Y, Anggriawan S, Chetan D et al (2014) Fate of the hypoplastic proximal aortic arch in infants undergoing repair for coarctation of the aorta through a left thoracotomy. Ann Thorac Surg 98(4):1386-1393

16. Karamlou T, Bernasconi A, Jaeggi E et al (2009) Factors associated with arch re-intervention and growth of the aortic arch after coarctation repair in neonates weighing less than $2.5 \mathrm{~kg}$. J Thorac Cardiovasc Surg 137(5):1163-1167

17. Gropler MRF, Marino BS, Carr MR et al (2019) Long-term outcomes of coarctation repair through left thoracotomy. Ann Thorac Surg 107(1):157-164
18. Mitchell ME (2017) Aortic coarctation repair: how i teach it. Ann Thorac Surg 104(2):377-381. https://doi.org/10.1016/j.athoracsur. 2017.03.084

19. Tulzer A, Mair R, Kreuzer M, Tulzer G (2016) Outcome of aortic arch reconstruction in infants with coarctation: importance of operative approach. J Thorac Cardiovasc Surg 152(6):1506-1513. e1

20. Ungerleider RM, Pasquali SK, Welke KF et al (2013) Contemporary patterns of surgery and outcomes for aortic coarctation: an analysis of the Society of Thoracic Surgeons Congenital Heart Surgery Database. J Thorac Cardiovasc Surg 145(1):150-158

21. Lee MGY, Allen SL, Koleff J et al (2018) Impact of arch reobstruction and early hypertension on late hypertension after coarctation repair. Eur J Cardio-Thorac Surg Off 53(3):531-537

22. Canniffe C, Ou P, Walsh K, Bonnet D, Celermajer D (2013) Hypertension after repair of aortic coarctation-a systematic review. Int J Cardiol 167(6):2456-2461

Publisher's Note Springer Nature remains neutral with regard to jurisdictional claims in published maps and institutional affiliations. 